\title{
NON-PULSATING STARS AND THE POPULATION I AND II INSTABILITY STRIPS
}

\author{
ARTHUR N. COX and JAMES E. TABOR \\ University of California, Los Alamos Scientific Laboratory, Los Alamos, N.M., U.S.A. \\ and \\ DAVID S. KING \\ University of New Mexico, Albuquerque, N.M., U.S.A.
}

\begin{abstract}
With specially computed detailed tables of equations of state and opacities, the instability strips for $\delta$ Scuti stars and Cepheids of population I and RR Lyrae and $\mathrm{W}$ Virginis stars of population II have been compared using the linear pulsation theory. Uncertainties in the observed strip locations and sometimes the mode of the observed pulsations do not allow high accuracy in fixing helium contents or the variables masses. Nevertheless, if masses close to those given by evolutionary theory are used, the helium content in population II objects is likely less than $Y=0.25$. A helium content of close to zero would put the theoretical blue edge of the instability strip to the red of the observed red edge, and have all the hotter stars which are in the strip as non-pulsating. For population I, $Y$ can be more than 0.3 , (more than 0.4 if half evolutionary masses are used), but if a given star has $Y$ less than about 0.2 (full mass) or 0.25 (half mass), it can appear in the dwarf and classical Cepheid strips as non-pulsating.
\end{abstract}

\section{DISCUSSION}

Breger: Dr Cox presented a diagram in which some stars were pulsating and some were not. This is another diagram like that for all these stars which have been tested for pulsation. Near the main sequence, there are $\delta$ Scuti variables and non-variables. Indeed two thirds of the stars in the instability strip do not show any pulsation in excess of one hundredth of a magnitude amplitude. If the amplitude of any of these at the time of observation were larger than a hundredth, I think they would have been detected. I think there's quite a bit of evidence for non-variable stars or stars with very little variation, unfortunately you cannot say which of these points are actually completely stable. In other words, the fact that you don't see this progression or wandering of the blue edge across this diagram or see a lot of variables in the middle and very few at the left, may purely be a reflection of the fact that you cannot distinguish between real non-variables and variables with very small amplitude. We have looked for pulsation in six population II stars - old disc population stars - with very high proper motion. None of the 6 stars showed any pulsation whatsoever. This is not a very big sample, and we are continuing our investigation to see whether we do in fact find more in our AI Velorum type stars, but so far it is very disheartening and there may indeed be quite a few non-variables in the instability strip amongst these population II, low mass, stars.

Schatzman: Dr Cox's results raise two problems. In the case of the stars near the main sequence, A. Baglin has shown that it was possible that gravitational separation of helium could take place, in which case the stars are not pulsating. The stars in which, due to mixing, helium separation has not taken place, are pulsating. This would explain the presence of the 2 types of stars in the same area. For the case of Cepheids this seems to be much more difficult because they have had a long evolutionary sequence and the separation should have taken place much before the giant phase. Off hand, I would say it is more difficult to explain.

Cox: I, of course, did not discuss the question of inhomogeneous stars. Let me point out that my paper surely does not easily explain variables and non-variables in the same cluster. You see, for a given cluster in which one would hope to have the same composition for all the stars, there should be only one blue edge corresponding to the one helium content. If there is helium separation from the helium 
ionization or driving region, then non-pulsation can occur. Rotation can prevent the separation making pulsators, but the observations seem to indicate rotation prevents, not enhances, pulsation. It may be that gravitational separation of helium increases the helium in the driving region making pulsation. Then rotation would cause mixing, a net helium depletion and no pulsation. Cepheids have not much rotation and pulsate nicely.

Bell: In connection with the diagram which Dr Breger showed, I'd like to ask you if you can calculate models which show a light amplitude of say 1 or 2 hundredths of a magnitude?

Cox: No, we have not done that, I said the work here was all linear theory so all I can do is get a blue edge. I just know that it is pulsationally unstable at infinitesimal amplitude. We should study these with a non-linear theory and we will, but non-linear theory is so complex, it takes a long time to do it.

Zahn: What is the $e$-folding time of the amplitude instability in Cepheids compared to the time they spend in the Cepheid strip?

Cox: Anywhere from 10 to $10^{6}$ periods the shorter growth times being for population II Cepheids and the longer growth times for $\delta$ Scuti stars. In any case, these times are always short compared to the time spent in the instability strip.

Bessell: Eggen and I observed a star last year which was variable over three nights of 3 hundredths of a magnitude; since then several others have observed it and found it not to vary. I have also observed several stars Danziger and Dickens suggested were variable and found no variations in them this year.

Cogan: What is the basis for stating that the position of the red edge of the instability strip is independent of chemical composition? You said that the radiative gradient was independent of chemical composition. Is this out of ignorance or do you have some reason for doing this?

Cox: I am told by some stellar structure people that they don't expect the onset of convection to be all that composition dependent, but unfortunately as we all admitted this morning, we don't really know exactly what it is about convection that causes the red edge.

Cogan: It is the onset of convection that causes the return to stability.

Cox: Yes. Alan Sandage suggested that if the helium moves the blue edge, it could also move the red edge and have the strip width constant. If reddening corrections and the conversion from color to effective temperature can be assured, the red edge might show a change with helium also. In this case we don't have adequate theory yet for the return to pulsational stability when there is convection.

Tayler: The results that have just been mentioned about the $\delta$ Scuti type stars, with the million period $e$-folding time are similar to the very high mass stars that I'm going to say something about to-morrow. In that case, since the higher harmonics are stable, you have a tremendous amount of time for interaction between the fundamental mode and the higher harmonics and it may be that although you think from a linear theory that you're going to get instability, you may get very little if the energy can be transferred by non-linear effects into the higher harmonics on a time short compared to the $e$-folding time. This is what we found in the high mass stars.

Cox: There is a problem about which harmonic theoretical blue edge corresponds to the observed blue edge for $\delta$ Scuti stars. All I know is that there is a blue edge defined for the fundamental, first harmonic, and second harmonic. I then inquire about what $Q$ value the observers say corresponds to the observed blue edge. The $Q$ value quoted is appropriate to the fundamental mode. Therefore I match the fundamental blue edge and get a reasonable helium content.

A further point is that if the helium fraction is lower than about 0.2 by mass, the big bang production value, then there is no pulsation for any star. These helium contents of 0.22 for population II stars and between 0.28 and 0.38 (or higher for less than evolutionary mass Cepheids) for population I stars seem reasonable. My paper is not able to get accurate helium contents due to the lack of precise observed blue edges.

Stobie: Surely this is a case where you could look at an external galaxy like the SMC where you can observe population I Cepheids and the question then is could the observations be of sufficient accuracy, could you detect any constant stars which are inside the strip?

Rodgers: You've got differential reddening that would foul the system.

Cox: The only way I have proposed to explain non-variable stars in the instability strip is to reduce their helium content which is causing the pulsation.

Iben: The only way you could explain away variables is to adopt a He abundance that's less than population II variables.

Rodgers: You had to go to a higher $Y$ value for population I didn't you?

Cox: Yes, you see, to get a Cepheid to pulsate in the instability strip, I have to have a $Y$ of about $0.3-$ 0.35 , or maybe if the mass is half the evolutionary mass, it's up to 0.5 . I need considerable helium enrichment to make population I stars pulsate. 